\title{
A new proof of the dual optimization problem and its application to the optimal material distribution of $\mathrm{SiC}$ /graphene composite
}

\author{
Ji-Huan He ${ }^{1}$ \\ ${ }^{1}$ National Engineering Laboratory for Modern Silk, College of Textile and Clothing Engineering, Soochow \\ University, Suzhou, China, \\ e-mail: hejihuan@suda.edu.cn
}

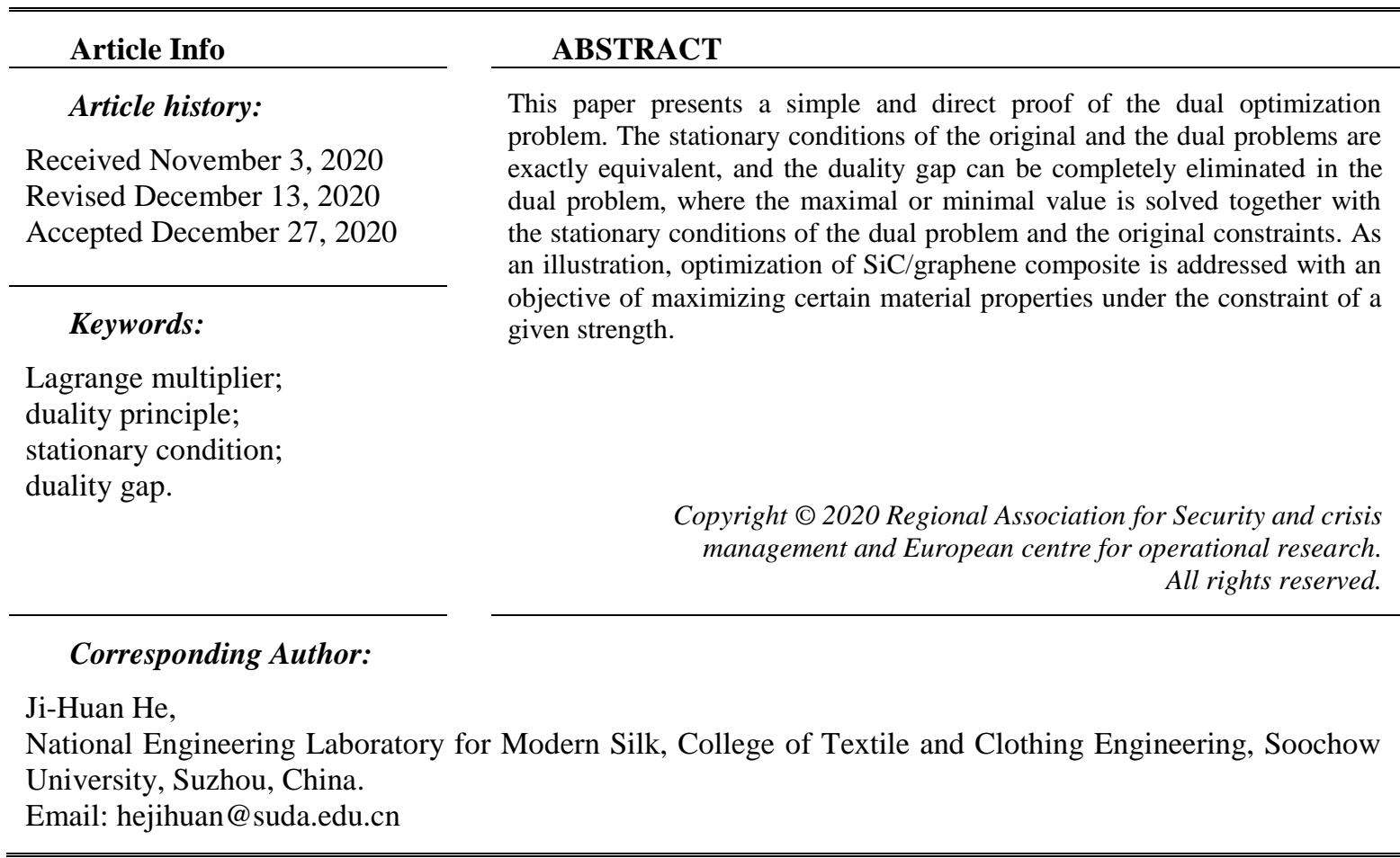

\section{Introduction}

Optimization is an important branch of mathematics that aims at finding the optimal solution (maximum or minimum) of a given problem and under given constraints. The problem is described by an objective function, the minimum or maximum of which is sought in a subspace of allowable solutions defined by the constraint functions. Optimization problems arise in all engineering disciplines. In particular, in mechanical engineering typical objectives include: 1) minimizing the energy necessary for various processes; 2) optimizing material usage, which implies minimizing the weight in various structural designs; 3) optimizing the material design so as to maximize the 'positive' properties such as strength, stiffness, conductivity, or minimize 'negative' properties like corrosion, abrasion, etc.

The final objective is to optimize the complete mechanical systems. In most cases, however, this is a cumbersome task, often involving multi-criteria optimization, whereby optimization procedures according to various criteria/objectives can even be conflicting. Various techniques have been developed for this purpose ranging from rigorous mathematical techniques such as derivative-based optimization and up to modern heuristic methods that aim at sufficiently good solutions rather than the absolute optimum. In many cases, it is also sufficient to optimize individual components of the system and then simply assemble the entire system. This is obviously a simplified approach compared to considering the system as a whole, but it produces satisfactory results on many occasions. 
Obviously, material design optimization plays an important role in mechanical engineering for an optimal material distribution or a minimal cost under various constraints (Hu, Yao, Huang, 2020).

The method of Lagrange multipliers as an approach has been extensively practiced in economics (Besada \& Mirás, 2002; Beviá \& Corchón, 2016; Caputo, 2001; Chatelain, 2000; Haeser \& de Melo, 2015; He, 2017; Juhl, 2004; Ponthiere, 2016; Weber, 1998), and its duality principle (Boyd \& Vandenberghe, 2004; Magno, et al., 2017) is also widely used in economics. For example, the original optimal problem is to maximize its profit of a factory, then its dual problem is to minimize the cost. Depending on the type of problem to be resolved, the same approach can be successfully applied in other disciplines, including mechanical engineering. In this short letter we give a direct proof of the dual optimization problem without using the Lagrange multiplier theory.

\section{The dual optimization problem}

To begin with the duality principle, we consider a standard optimization problem

Maximize $F\left(x_{1}, x_{2}\right)$

Subject to $G\left(x_{1}, x_{2}\right)=0$

The stationary condition is to maximize $F$, this requires (He, 2008; Qin \& Ge, 2010)

$d F\left(x_{1}, x_{2}\right)=\frac{\partial F}{\partial x_{1}} d x_{1}+\frac{\partial F}{\partial x_{2}} d x_{2}=0$

Hereby $d x_{1}$ and $d x_{2}$ are not independent, their variations should follow the requirement given in Eq. (2), that is

$d G\left(x_{1}, x_{2}\right)=\frac{\partial G}{\partial x_{1}} d x_{1}+\frac{\partial G}{\partial x_{2}} d x_{2}=0$

From Eqs.(3) and (4), we have the following stationary condition:

$\left|\begin{array}{ll}\frac{\partial F}{\partial x_{1}} & \frac{\partial F}{\partial x_{2}} \\ \frac{\partial G}{\partial x_{1}} & \frac{\partial G}{\partial x_{2}}\end{array}\right|=0$

Eq. (5) can also be derived by using He's brackets (He,2008; Qin \& Ge, 2010). Now we give the dual optimization problem:

Minimize $G\left(x_{1}, x_{2}\right)$

Subject to $F\left(x_{1}, x_{2}\right)=F_{\max }$

Remark 1 .

In order to eliminate the duality gap (Boyd \& Vandenberghe, 2004), $F\left(x_{1}, x_{2}\right)=F_{\max }$ in stead of $F\left(x_{1}, x_{2}\right)=F_{0}$, where $F_{\max }$ the optimal value, $F_{0}$ is a constant. 2010)

The stationary condition of the dual problem can be readily obtained, which is(He,2008; Qin \& Ge,

$\left|\begin{array}{ll}\frac{\partial G}{\partial x_{1}} & \frac{\partial G}{\partial x_{2}} \\ \frac{\partial F}{\partial x_{1}} & \frac{\partial F}{\partial x_{2}}\end{array}\right|=0$

Eq. (8) is equivalent to Eq. (5), and the optimal solution is obtained by solving Eq. (8) and Eq. (7) simultaneously.

For multiple constraints, we can obtain a similar result. Consider the following equality-constrained problem:

Reports in Mechanical Engineering, Vol. 1, No. 1, 2020: 187 - 191 
Minimize $F\left(x_{1}, x_{2}, x_{3}\right)$

Subject to $G\left(x_{1}, x_{2}, x_{3}\right)=0, H\left(x_{1}, x_{2}, x_{3}\right)=0$

The stationary condition is (He, 2008 ; Qin \& Ge, 2010)

$\left|\begin{array}{lll}F_{x_{1}} & F_{x_{2}} & F_{x_{3}} \\ G_{x_{1}} & G_{x_{2}} & G_{x_{3}} \\ H_{x_{1}} & H_{x_{2}} & H_{x_{3}}\end{array}\right|=0$

Its dual optimization problem is

Maximize $G\left(x_{1}, x_{2}, x_{3}\right)$

Subject to $F\left(x_{1}, x_{2}, x_{3}\right)=F_{\text {min }}, H\left(x_{1}, x_{2}, x_{3}\right)=0$

Remark 2.

Hereby $F_{\min }$ is the optimal value of the original optimal problem, no duality gap occurs.

The stationary condition of the dual problem is (He,2008; Qin \& Ge, 2010)

$\left|\begin{array}{lll}G_{x_{1}} & G_{x_{2}} & G_{x_{3}} \\ F_{x_{1}} & F_{x_{2}} & F_{x_{3}} \\ H_{x_{1}} & H_{x_{2}} & H_{x_{3}}\end{array}\right|=0$

Eq. (15) is equivalent to Eq. (12), and the optimal solution is obtained by solving Eqs. (15) and (14) and the first constraint of Eq. (10) simultaneously.

An example is given below (Izmailov \& Solodov, 2009)

Minimize $F\left(x_{1}, x_{2}, x_{3}\right)=x_{1}^{2}-x_{2}^{2}+x_{3}^{2}$

Subject to $G\left(x_{1}, x_{2}, x_{3}\right)=x_{1}^{2}+x_{2}^{2}-x_{3}^{2}, H\left(x_{1}, x_{2}, x_{3}\right)=x_{1} x_{3}$

The stationary condition is(He,2008; Qin \& Ge, 2010)

$\left|\begin{array}{lll}F_{x_{1}} & F_{x_{2}} & F_{x_{3}} \\ G_{x_{1}} & G_{x_{2}} & G_{x_{3}} \\ H_{x_{1}} & H_{x_{2}} & H_{x_{3}}\end{array}\right|=\left|\begin{array}{ccc}2 x_{1} & -2 x_{2} & 2 x_{3} \\ 2 x_{1} & 2 x_{2} & -2 x_{3} \\ x_{3} & 0 & x_{1}\end{array}\right|=4 x_{1}^{2} x_{2}+4 x_{3}^{2} x_{2}-4 x_{3}^{2} x_{2}+4 x_{1}^{2} x_{2}=8 x_{1}^{2} x_{2}=0$

Solving Eqs. (17) and (18) results in the optimal solution $\left(x_{1}^{*}, x_{2}^{*}\right)=(0,0)$.

Its dual optimization problem is

Maximize $G\left(x_{1}, x_{2}, x_{3}\right)=x_{1}^{2}+x_{2}^{2}-x_{3}^{2}$

Subject to $F\left(x_{1}, x_{2}, x_{3}\right)=x_{1}^{2}-x_{2}^{2}+x_{3}^{2}=F_{\text {min }}, H\left(x_{1}, x_{2}, x_{3}\right)=x_{1} x_{3}$

The stationary equation is

$\left|\begin{array}{lll}G_{x_{1}} & G_{x_{2}} & G_{x_{3}} \\ F_{x_{1}} & F_{x_{2}} & F_{x_{3}} \\ H_{x_{1}} & H_{x_{2}} & H_{x_{3}}\end{array}\right|=\left|\begin{array}{ccc}2 x_{1} & 2 x_{2} & -2 x_{3} \\ 2 x_{1} & -2 x_{2} & 2 x_{3} \\ x_{3} & 0 & x_{1}\end{array}\right|=-8 x_{1}^{2} x_{2}=0$

The optimal solution can be easily obtained, which is $\left(x_{1}^{*}, x_{2}^{*}, F_{\min }\right)=(0,0,0)$. 


\section{An application}

As an application, we consider a SiC/graphene composite (Zuo \& Liu, 2021). We assume that the concentration of graphene is $\varphi$, according to Zuo-Liu's formulation, the composite's conductivity can be written as (Zuo \& Liu, 2021)

$C=C_{0}+k \varphi^{1.25}$

where $\mathrm{C}$ is the conductivity of the composite, $C_{0}$ is the carborundum's conductivity, $\mathrm{k}$ is a constant.

The composite's stress, according to o Zuo-Liu's formulation (Zuo \&Liu, 2021)

$$
\sigma=\frac{\sigma_{0}-a \varphi^{2 / 3}}{1+b \varphi^{2 / 3}}
$$

where $\sigma_{0}$ is the stress without graphene. We assume that $\sigma_{0}$ and $C_{0}$ have the following linear relationship

$$
C_{0}=\lambda \sigma_{0}
$$

The optimal problem is

Maximize $C=\lambda \sigma_{0}+k \varphi^{1.25}$

Subject to $\frac{\sigma_{0}-a \varphi^{2 / 3}}{1+b \varphi^{2 / 3}}=\bar{\sigma}$

Its stationary condition is

$\left|\begin{array}{cc}\lambda & 1.25 k \varphi^{0.25} \\ \frac{\sigma_{0}}{1+b \varphi^{2 / 3}} & \frac{-\frac{2}{3} a \varphi^{-1 / 3}\left(1+b \varphi^{2 / 3}\right)-\frac{2}{3} b \varphi^{-1 / 3}\left(\sigma_{0}-a \varphi^{2 / 3}\right)}{\left(1+b \varphi^{2 / 3}\right)^{2}}\end{array}\right|=0$

Its dual optimization problem is

Maximize $\sigma=\frac{\sigma_{0}-a \varphi^{2 / 3}}{1+b \varphi^{2 / 3}}$

Subject to $\lambda \sigma_{0}+k \varphi^{1.25}=\mathrm{C}_{\max }$

For given parameters involved in Eqs. (24) and (25), the optimal value can be easily solved.

\section{Conclusions}

In this letter, optimization is addressed as an important method used in engineering to produce best or nearly best results under given circumstances. A direct proof of the duality principle in optimization is given. As the concept of the dual optimization is widely adopted in economics and engineering, this paper gives a strictly mathematical foundation for the dual optimization. Furthermore, the duality gap (Boyd \& Vandenberghe, 2004) can be completely eliminated. This important property is successfully demonstrated in the task of optimizing material properties of a SiC/graphene composite.

\section{References}

Besada, M., \& Mirás,M. (2002). A note on Lagrange multipliers in the multiple constraint case, Economics Letters, 75(1), 141-145.

Beviá, C., \& Corchón, L. C. (2017). Growth in Illyria: The role of meritocracy in the accumulation of human capital, Mathematical Social Sciences, 90, 182-190.

Boyd, S. P., \& Vandenberghe, L. (2004). Convex Optimization, Cambridge University Press. 
Caputo, M. R. (2001). Further results on Lagrange multipliers with several binding constraints, Economics Letters, 70(3), 335-3404.

Chatelain, J.-B. (2000). Explicit Lagrange multiplier for firms facing a debt ceiling constraint, Economics Letters, 67(2), 153-158.

Haeser, G., \& de Melo, V. V. (2015). Convergence detection for optimization algorithms: Approximate-KKT stopping criterion when Lagrange multipliers are not available, Operations Research Letters 43, 484-488.

He, J.H. (2006). Some asymptotic methods for strongly nonlinear equations , International Journal of Modern Physics B, 20(10),1141-1199.

He, J.H. (2008). An elementary introduction to recently developed asymptotic methods and nanomechanics in textile engineering, International Journal of Modern Physics B, 22(21), 3487-3578.

He, J.H. (2017). A tutorial and heuristic review on Lagrange multiplier for optimal problems, Nonlinear Science Letters A, 8, 121-148.

Hu, J., Yao, S., \& Huang, X. (2020). Topology optimization of dynamic acoustic-mechanical structures using the ersatz material model, Computer Methods in Applied Mechanics and Engineering. 372, Article Number: 113387.

Izmailov, A.F. \& Solodov, M.V. (2009). Examples of dual behaviour of Newton-type methods on optimization problems with degenerate constraints, Computational Optimization and Applications 42, 231 264.

Juhl, T. (2004). A Lagrange multiplier stationarity test using covariates, Economics Letters, 85(3), 321-3263.

Magno, G. L. L., Ferrante, M., \& De Cantis, S. (2017). A new index for measuring seasonality: A transportation cost approach, Mathematical Social Sciences, 88, 55-65.

Ponthiere, G. (2016). Pollution, unequal lifetimes and fairness, Mathematical Social Sciences, 82, 49-64

Qin, S.T., \& Ge, Y. (2010). A Novel Approach to Markowitz Portfolio Model without Using Lagrange Multipliers, Int. J. Nonlin. Sci. Num., 11, s331-s334.

Weber, C.E. (1998). A note on Lagrange multipliers with several binding constraints. Economics Letters, 59(1), 71-752.

Zuo, Y.T., \& Liu, H.J. (2021). A fractal rheological model for sic paste using a fractal derivative, Journal of Applied and Computational Mechanics, 7, 13-18. 